Let $r=\epsilon\left(h^{\prime}+c j\right)$, then $r=\epsilon\left(h^{\prime}\right) \cdot \epsilon(c j)=r^{\prime} \epsilon(c j)$, and from (5.2), we have

$$
\begin{aligned}
\sigma(r) & =\sigma\left(r^{\prime}\right) \cdot \sigma(\epsilon(c j))=\sigma\left(r^{\prime}\right) \cdot \epsilon(\sigma(c j)) \\
& =\sigma\left(r^{\prime}\right) \epsilon(c j)=s r^{\prime} \cdot \epsilon(c j)=s r .
\end{aligned}
$$

Moreover, (6.3) and (6.4) tell us that $r$ is regular in G. Lemma 2 is thus proved.

\title{
REFERENCES
}

1. E. Cartan, La géometrie des groupes simples, Ann. Mat. Pura Appl. 4 (1927), 209-256.

2. M. Goto, $A$ theorem on compact semi-simple groups, J. Math. Soc. Japan 1 (1948), 270-273.

3. K. Shoda, Einige Satze ilber Matrizen, Japan J. Math. 13 (1937, 361-365).

4. R. C. Thompson, Commutators of matrices. III, Abstracts 565-16, Notices Amer. Math. Soc. 7 (1960), 194.

5. B. Kostant, The principal 3-dimensional subgroup and the Betti numbers of a complex simple Lie group, Amer. J. Math. 81 (1959), 973-1032.

NORTHWESTERN UNIVERSITY

\section{SOME INTEGRAL FORMULAS FOR THE COMPLETE ELLIPTIC INTEGRALS OF THE FIRST AND SECOND KIND}

\author{
L. CARLITZ ${ }^{1}$
}

In the usual notation let

$$
\begin{aligned}
& K(k)=\frac{\pi}{2} \sum_{0}^{\infty} \frac{\left(\frac{1}{2}\right)_{n}\left(\frac{1}{2}\right)_{n}}{n ! n !} k^{2 n} \\
& E(k)=\frac{\pi}{2} \sum_{0}^{\infty} \frac{\left(-\frac{1}{2}\right)_{n}\left(\frac{1}{2}\right)_{n}}{n ! n !} k^{2 n}
\end{aligned}
$$

denote the complete elliptic integrals of the first and second kind, respectively. It is easily verified that (2) implies

Received by the editors September 26, 1961 and, in revised form, November 21, 1961.

${ }^{1}$ Supported in part by National Science Foundation grant G-16485. 


$$
\frac{E(k)}{1-k^{2}}=\frac{\pi}{2} \sum_{0}^{\infty} \frac{\left(\frac{1}{2}\right)_{n}\left(\frac{3}{2}\right)_{n}}{n ! n !} k^{2 n} .
$$

We now consider the integral

$$
\int_{0}^{\pi / 2} \frac{E(i k \sin \theta)}{1+k^{2} \sin ^{2} \theta} \frac{E(k \cos \theta)}{1-k^{2} \cos ^{2} \theta} \sin \theta \cos \theta d \theta .
$$

Making use of (3) this becomes

$$
\begin{aligned}
\frac{\pi^{2}}{4} \sum_{r=0}^{\infty}(-1)^{r} \frac{\left(\frac{1}{2}\right)_{r}\left(\frac{3}{2}\right)_{r}}{r ! r !} k^{2 r} \sum_{s=0}^{\infty} \frac{\left(\frac{1}{2}\right)_{s}\left(\frac{3}{2}\right)_{s}}{s ! s !} k^{2 s} \\
\quad \int_{0}^{\pi / 2} \sin ^{2 r+1} \theta \cos ^{2 s+1} \theta d \theta \\
=\frac{\pi^{2}}{8} \sum_{r, s=0}^{\infty}(-1)^{r} \frac{\left(\frac{1}{2}\right)_{r}\left(\frac{3}{2}\right)_{r}}{r ! r !} \frac{\left(\frac{1}{2}\right)_{s}\left(\frac{3}{2}\right)_{s}}{s ! s !} k^{2 r+2 s} \frac{r ! s !}{(r+s+1) !}
\end{aligned}
$$

(5)

$$
\begin{aligned}
& =\frac{\pi^{2}}{8} \sum_{n=0}^{\infty} \frac{k^{2 n}}{(n+1) !} \sum_{r=0}^{n}(-1)^{r} \frac{\left(\frac{1}{2}\right)_{r}\left(\frac{3}{2}\right)_{r}\left(\frac{1}{2}\right)_{n-r}\left(\frac{3}{2}\right)_{n \rightarrow r}}{r !(n-r) !} \\
& =\frac{\pi^{2}}{8} \sum_{n=0}^{\infty} \frac{\left(\frac{1}{2}\right)_{n}\left(\frac{3}{2}\right)_{n}}{n !(n+1) !} k^{2 n} \sum_{r=0}^{n} \frac{(-n)_{r}\left(\frac{1}{2}\right)_{r}\left(\frac{3}{2}\right)_{r}}{r !\left(\frac{1}{2}-n\right)_{r}\left(-\frac{1}{2}-n\right)_{r}} \\
& =\frac{\pi^{2}}{8} \sum_{n=0}^{\infty} \frac{\left(\frac{1}{2}\right)_{n}\left(\frac{3}{2}\right)_{n}}{n !(n+1) !} k^{2 n} \cdot{ }_{3} F_{2}\left[\begin{array}{l}
-n, \frac{1}{2}, \frac{3}{2} ; \\
\frac{1}{2}-n,-\frac{1}{2}-n
\end{array}\right] .
\end{aligned}
$$

Clearly the ${ }_{3} F_{2}$ vanishes for odd $n$; for even $n$ we apply Dixon's theorem $[1$, p. 13]:

(6) ${ }_{3} F_{2}\left[\begin{array}{ccc}-2 n, & \alpha, & \beta ; \\ 1-\alpha-2 n, & 1-\beta-2 n\end{array}\right]=\frac{(2 n) !(\alpha)_{n}(\beta)_{n}(\alpha+\beta)_{2 n}}{n !(\alpha+\beta)_{n}(\alpha)_{2 n}(\beta)_{2 n}}$.

In particular 
1962] COMPLETE ELLIPTIC INTEGRALS OF FIRST AND SECOND KIND

$$
\begin{aligned}
{ }_{3} F_{2}\left[\begin{array}{c}
-2 n, \frac{1}{2}, \frac{3}{2} ; \\
\frac{1}{2}-2 n,-\frac{1}{2}-2 n
\end{array}\right]=\frac{(2 n) !}{n !} \frac{\left(\frac{1}{2}\right)_{n}\left(\frac{3}{2}\right)_{n}}{(n+1) !} \frac{(2 n+1) !}{\left(\frac{1}{2}\right)_{2 n}\left(\frac{3}{2}\right)_{2 n}} \\
=\frac{(2 n) !}{n !} \frac{(2 n+1) !}{\left(\frac{1}{2}\right)_{n}} \frac{\left(\frac{3}{2}\right)_{n}}{\left(\frac{1}{2}\right)_{2 n}}, \frac{\left(\frac{3}{2}\right)_{2 n}}{}
\end{aligned}
$$

so that (5) becomes

$$
\begin{aligned}
\frac{\pi^{2}}{8} \sum_{n=0}^{\infty} \frac{\left(\frac{1}{2}\right)_{2 n}\left(\frac{3}{2}\right)_{2 n}}{(2 n) !(2 n+1) !} \frac{(2 n) !(2 n+1) !\left(\frac{1}{2}\right)_{n}\left(\frac{3}{2}\right)_{n}}{n !(n+1) !\left(\frac{1}{2}\right)_{2 n}\left(\frac{3}{2}\right)_{2 n}} k^{4 n} \\
=\frac{\pi^{2}}{8} \sum_{n=0}^{\infty} \frac{\left(\frac{1}{2}\right)_{n}\left(\frac{3}{2}\right)_{n}}{n !(n+1) !} k^{4 n} .
\end{aligned}
$$

Since by (1) and (2)

$$
\begin{aligned}
K(k)-E(k) & =\frac{\pi}{2} \sum_{n=0}^{\infty}\left(\frac{\left(\frac{1}{2}\right)_{n}\left(\frac{1}{2}\right)_{n}}{n ! n !}-\frac{\left(-\frac{1}{2}\right)_{n}\left(\frac{1}{2}\right)_{n}}{n ! n !}\right)^{2 n} \\
& =\frac{\pi}{2} \sum_{n=1}^{\infty} \frac{\left(\frac{1}{2}\right)_{n}\left(\frac{1}{2}\right)_{n-1}}{n !(n-1) !} k^{2 n} \\
& =\frac{\pi}{4} \sum_{n=0}^{\infty} \frac{\left(\frac{1}{2}\right)_{n}\left(\frac{3}{2}\right)_{n}}{n !(n+1) !} k^{2 n+2}
\end{aligned}
$$

it follows that

$$
\begin{gathered}
\int_{0}^{\pi / 2} E(i k \sin \theta) E(k \cos \theta) \frac{\sin \theta \cos \theta d \theta}{\left(1+k^{2} \sin ^{2} \theta\right)\left(1-k^{2} \cos ^{2} \theta\right)} \\
=\frac{\pi}{2 k^{4}}\left(K\left(k^{2}\right)-E\left(k^{2}\right)\right) .
\end{gathered}
$$


In the next place we have

$\int_{0}^{\pi / 2} K(i k \sin \theta) K(k \cos \theta) \sin \theta \cos \theta d \theta$

$$
\begin{aligned}
& =\frac{\pi^{2}}{8} \sum_{r, s=0}^{\infty}(-1)^{r} \frac{\left(\frac{1}{2}\right)_{r}\left(\frac{1}{2}\right)_{r}}{r ! r !} \frac{\left(\frac{1}{2}\right)_{s}\left(\frac{1}{2}\right)_{s}}{s ! s !} \frac{r ! s !}{(r+s+1) !} k^{2 r+2 s} \\
& =\frac{\pi^{2}}{8} \sum_{n=0}^{\infty} \frac{\left(\frac{1}{2}\right)_{n}\left(\frac{1}{2}\right)_{n}}{n !(n+1) !} k^{2 n} \sum_{r=0}^{n} \frac{(-n)_{r}\left(\frac{1}{2}\right)_{r}\left(\frac{1}{2}\right)_{r}}{r !\left(\frac{1}{2}-n\right)_{r}\left(\frac{1}{2}-n\right)_{r} .}
\end{aligned}
$$

Applying (6) this reduces to

$$
\frac{\pi^{2}}{8} \sum_{n=0}^{\infty} \frac{\left(\frac{1}{2}\right)_{n}\left(\frac{1}{2}\right)_{n}}{n ! n !} \frac{k^{4 n}}{2 n+1}
$$

so that

(8)

$$
\begin{aligned}
\int_{0}^{\pi / 2} K(i k \sin \theta) K(k \cos \theta) \sin \theta \cos \theta d \theta & =\frac{\pi^{2}}{8} \sum_{n=0}^{\infty} \frac{\left(\frac{1}{2}\right)_{n}\left(\frac{1}{2}\right)_{n}}{n ! n !} \frac{k^{4 n}}{2 n+1} \\
& =\frac{\pi}{4 k^{2}} \int_{0}^{k} K(x) d x .
\end{aligned}
$$

In exactly the same way we find that

(9)

$$
\begin{aligned}
& \int_{0}^{\pi / 2} E(i k \sin \theta) E(k \cos \theta) \sin \theta \cos \theta d \theta \\
& =\frac{\pi^{2}}{16}+\frac{\pi^{2}}{16} \sum_{n=0}^{\infty} \frac{\left(-\frac{1}{2}\right)_{n}\left(\frac{1}{2}\right)_{n}}{n ! n !} \frac{k^{4 n}}{2 n+1}=\frac{\pi^{2}}{16}+\frac{\pi}{8 k^{2}} \int_{0}^{k} E(x) d x .
\end{aligned}
$$

Since

$$
\frac{d}{d k}(k E(k))=2 E(k)-K(k),
$$

it follows from (8) and (9) that 


$$
\begin{aligned}
\int_{0}^{\pi / 2} & \{4 E(i k \sin \theta) E(k \cos \theta)-K(i k \sin \theta) K(k \cos \theta)\} \sin \theta \cos \theta d \theta \\
= & \frac{\pi^{2}}{4}+\frac{\pi}{4} E\left(k^{2}\right)
\end{aligned}
$$

In exactly the same way one can prove the following more general results. Put

$$
\begin{aligned}
& A=\int_{0}^{\pi / 2} F\left[a, b ; c ;-x \sin ^{2} \theta\right] F\left[a, b ; d ; x \cos ^{2} \theta\right] \sin ^{2 c-1} \theta \cos ^{2 d-1} \theta d \theta \\
& B=\int_{0}^{\pi / 2} F\left[a,-a ; c ;-x \sin ^{2} \theta\right] F\left[a,-a ; d ; x \cos ^{2} \theta\right] \sin ^{2 c-1} \theta \cos ^{2 d-1} \theta d \theta .
\end{aligned}
$$

Then

$$
\begin{aligned}
& A=\frac{\Gamma(c) \Gamma(d)}{2 \Gamma(c+d)}{ }_{4} F_{8}\left[\begin{array}{c}
a, b, \frac{1}{2}(a+b), \frac{1}{2}(a+b+1) ; \\
a+b, \frac{1}{2}(c+d), \frac{1}{2}(c+d+1) ;
\end{array}\right], \\
& B=\frac{\Gamma(c) \Gamma(d)}{4 \Gamma(c+d)}\left\{1+{ }_{8} F_{2}\left[\begin{array}{c}
\frac{1}{2}, a,-a ; \\
\frac{1}{2}(c+d), \frac{1}{2}(c+d+1) ;
\end{array}\right]\right\} .
\end{aligned}
$$

If in the evaluation of $A$ we take $a=1 / 2, b=3 / 2, c=d=1$ and replace $x$ by $k$ we get (7); if we take $a=b=1 / 2, c=d=1$ we get (8). Similarly if in the evaluation of $B$ we take $a=1 / 2, c=d=1$ and replace $x$ by $k$ we get (9).

\section{REFERENCE}

1. W. N. Bailey, Generalized hypergeometric series, Cambridge Univ. Press, Cambridge, 1935.

DURe UnIVERSITY 